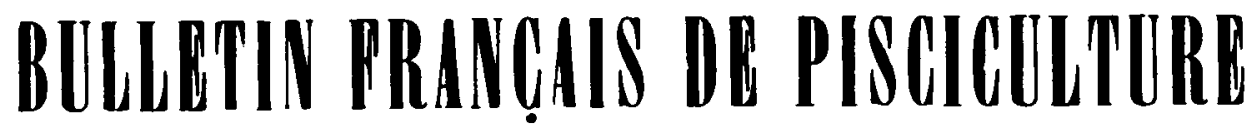 \\ VINGT-QUATRIÈE ANNEE \\ No 183 \\ OCTOBRE-DECEMBRE 1951
}

\section{OBSERVATIONS SUR LA PECCHE DE L'ESTURGEON (ACIPENSER STURIO L.) DANS LE GUADALQUIVIR DURANT L'ANNÉE $1950^{(1)}$}

L'Esturgeon commun (Acipenser sturio) était, autrefois, abondant dans presque tous les fleuves de France et dans leurs principaux affluents. Actuellement, si on le rencontre accidentellement dans la Seine, la Loire, l'Adour ainsi que dans la Saône et dans le Doubs, il n'est réellement abondant que dans le Rhône et surtout dans la Garonne, où la production du caviar porte sur environ 3 tonnes par an si l'on se refère à l'article récent de MM. DARLET et G. Prioux (2).

En France, des mesures ont été prises en 1950, en vue d'assurer une protection plus complète de ce magnifique poisson : en effet, en accord avec les Services de la Marine marchande, un décret du 14 Septembre 1950 a fixé désormais la période d'interdiction de la pêche de l'Esturgeon du 1 er Juillet au 31 Décembre, tenu compte du fait que, pour sauvegarder l'espèce, il importe, tout en assurant bien entendu la protection des géniteurs (au surplus très prolifiques) durant une partie de leur fraie, d'empêcher que les jeunes Esturgeons, avenir de l'espèce, ne soient capturés dans les estuaires avant qu'ils ne gagnent la mer.

En Espagne, si l'Esturgeon se rencontre dans différents cours d'eau tels que le Guadiana, l'Ebre, etc., il est surtout abondant dans le Guadalquivir où sa pêche est réellement aménagée. Sur cette pêche, M. Francisco Velez Sото, Ingénieur des Eaux et Forêts, attaché au Service de la Pêche fluviale espagnole, a fait un certain nombre d'observations, particulièrement intéressantes, dans un article que nous reproduisons à peu près intégralement ci-après, en nous permettant, à cette occasion, d'adresser à notre collegue nos bien vifs compliments..

\section{René CHARPY,}

Conservateur des Eaux et Forêts,

Chef du Service des Relations avec les Pêcheurs.

(1) D'après : " Observaciones sobre la pesca del esturion en el rio Guadalquivir durante el ano 1950 " par Francisco Velez Soto, Ingénieur des Eaux et Forêts, attaché au Service de la Pêche fluviale Espagnole. Revue Montes, Janvier-Février 1951, no 37, p. 33 à 40.

(2) L'Esturgeon et le Caviar Français, par MM. Darlet et G. Prioux, Bulletin français de Pisciculture, no 158 , Juillet-Septembre 1950. 
Malgré les appels répétés lancés par divers auteurs, tant dans les livres que les revues, pour dénoncer le danger de disparition qui menace l'une des espèces les plus précieuses de notre faune fluviale, les milieux scientifiques espagnols n'ont jamais attaché beaucoup d'importance à l'étude de l'Esturgeon.

Les causes naturelles de diminution de ce poisson ont été indiquées par Lazano : perte de vitalité de l'espèce au cours d'une évolution déjà fort longue (l'origine du groupe des Ganoïdes remonte au Dévonien), organisation et facultés ne donnant guère à ce poisson de solides possibilités dans la lutte pour la vie. Mais à ces causes, il faut ajouter celles qui proviennent de l'homme : obstacles créés sur le parcours de l'Esturgeon lors de sa remontée dans nos cours d'eau, modifications apportées aux lits de ces cours d'eau, etc. Tout cela influe grandement lorsqu'il s'agit d'un poisson d'une organisation aussi primitive et qui possède d'aussi faibles facultés d'adaptation à un nouveau genre de vie.

Des représentants de l'espèce ont été capturés dans plusieurs rivières espagnoles (Guadiana, Ebre, etc.), mais dans aucune de celles-ci la péche n'a été organisée comme elle l'est dans le Guadalquivir, peut-être parce que cette rivière est la seule d'Espagne où la pêche de l'Esturgeon présente assez d'importance pour faire l'objet d'un profit industriel. L'utilisation des cufs d'Esturgeon pour la fabrication du Caviar et le prix élevé atteint par les oufs, fait que la pêche de ce poisson est d'un excellent rendement. Aussi serait-il désirable de le protéger, surtout lorsque l'on sait que cette espèce est appelée à disparaître finalement de notre faune.

Ce danger est-il imminent? Serait-il possible de l'éviter en protégeant l'espèce ? Répondre, tout au moins partiellement, à ces questions, tel est le but de la présente note, bien que nous ne possédions pas à l'heure actuelle de données suffisantes pour permettre des affirmations catégoriques en la matière.

En ce faisant, M. l'Ingénieur Francisco Velez Soto, a tenu à mettre à profit et à poursuivre pour le grand bénéfice de l'espèce, les études consciencieuses réalisées de 1931 à 1944 par le docteur T. E. A. Classen, études qui ont fait l'objet, de la part de celui-ci, mort depuis, d'un travail intitulé "Étude bio-statistique de l'Esturgeon du Guadalquivir ".

L'Esturgeon est un poisson anadrome. On sait peu de choses sur sa vie en mer. Il vit sans aucun doute dans des fonds où il rencontre en abondance la nourriture dont il a besoin pour atteindre ses dimensions énormes.

A 6 ou 8 ans, selon Classen, ses organes sexuels commencent à se développer, mais ils n'atteindraient leur maturité qu'à 11 ans chez les mâles, 14 ans chez les femelles.

C'est alors que les Esturgeons se rapprochent des côtes, attirés par "l'odeur de l'eau douce ", et remontent nos cours d'eau, à la recherche des fonds sableux et propres où ils pourront, dans un bon courant, donner libre cours à leurs instincts génésiques.

Une fois la fraie accomplie, les géniteurs retournent à la mer pour remonter à nouveau dans nos cours d'eau l'année suivante, du moins pour les mâles, ou deux ans après, pour les femelles. 
Ces observations faites par Classen dans la Guadalquivir corroborent, au surplus, celles de Dershaun, Ildin, Nedoshivin, etc., dans la mer Caspienne.

Sauf quelques rares exemplaires de jeunes Esturgeons qui y auraient été entraînés sous les poussées des autres, on ne capture dans la rivière que des individus adultes dont la taille varie entre 140 et 240 centimètres pour les femelles, correspondant à des poids variant entre 15 et $85 \mathrm{~kg}$., et entre 100 et 190 centimètres pour les mâles qui, en poids, atteignent parfois jusqu'à $45 \mathrm{~kg}$. Mais les dimensions les plus fréquentes des femelles oscillent entre 160 et 220 centimètres avec des poids de 25 à $55 \mathrm{~kg}$.; celles des mâles entre 130 et 170 centimètres avec des poids de 10 à $25 \mathrm{~kg}$.

Une fois la fraie accomplie (au Printemps) les oufs restent fixés au fonds de la rivière et les jeunes Esturgeons éclosent vers le quatrième ou cinquième jour. Ils mesurent environ 9 millimètres de longueur. Leur vésicule vitelline, qui est fortement colorée, se résorbe au cours des cinq ou six premiers jours de leur vie. Les jeunes Esturgeons commencent alors à s'alimenter avec des éléments du plancton et, plus tard, avec des animaux supérieurs.

Ils croissent rapidement pour atteindre 4 centimètres au bout d'un mois et 35 centimètres à l'automne. Durant tout ce temps, ils descendent la rivière jusqu'à ce qu'ils arrivent à la partie basse, où ils s'arrêtent pour s'accoutumer à l'eau salée.

Lorsqu'ils sont âgés d'un an ou d'un an et demi, ils pénètrent enfin dans la mer, mais sans s'éloigner au début de l'embouchure des cours d'eau.

Il était nécessaire de rappeler ces quelques considérations qui nous aideront à mieux comprendre ce qui va suivre.

Ainsi que rappelé au début de la présente note, la diminution de l'Esturgeon provient de causes naturelles, mais aussi de l'action humaine. Pour le Guadalquivir, cette action humaine présente deux aspects principaux : modifications apportées au lit même de la rivière, coupure du Guadalquivir par le barrage d'Alcala del Rio.

Jusqu'en 1932, les Esturgeons se pêchaient à Alcala del Rio et même au-dessus, à Lora del Rio, en dehors de la zone d'influence des marées. Mais cette année-là, la construction du barrage d'Alcala del Rio, entraîna l'impossibilité, pour les Esturgeons, d'accéder à leurs frayères naturelles et les obligea à déposer leurs œufs en aval du barrage, là où se font encore sentir les effets des marées et où l'eau n'est pas tout à fait douce. Les Esturgeons n'ont, en effet, la faculté de franchir le barrage que lorsqu'il est nécessaire, en cas d'excès d'eau, d'en ouvrir les vannes. Il est d'ailleurs assez curieux d'examiner les efforts faits par les Esturgeons pour remonter le courant qui sort des turbines où beaucoup se mutilent. Il y a bien une échelle à poissons dans le barrage, mais les Esturgeons sont incapables de la remonter, surtout les femelles, ceci en raison de leurs énormes dimensions et aussi du poids de leurs ovaires qui les oblige à se trainer sur le fonds de la rivière.

L'Esturgeon est donc obligé de frayer, soit à l'aval du barrage, soit au pied de celui-ci, soit encore plus bas, à La Algaba, et aussi de s'adapter 
aux eaux quelque peu salées de ces lieux. Au surplus, en raison des sécheresses persistantes de ces dernières années et aussi de l'utilisation presque totale des eaux du Guadalquivir pour l'irrigation, l'Esturgeon n'a même pas pu arriver jusqu'à Alcala et a été dans l'obligation de s'arrêter en pleine zone des marées.

Donc, perte pour l'Esturgeon de frayères naturelles et obligation pour lui de frayer dans des eaux salées, en dehors de son milieu naturel.

Passons maintenant à l'étude des pêches réalisées en 1950 dans le Guadalquivir, en vue d'en tirer des conséquences sur l'influence qu'a pu avoir le barrage d'Alcala sur l'avenir de l'espèce.

Nous devons toutefois, au préalable, résumer brièvement les recherches effectuées par Classen en vue de déterminer les relations qui existent entre l'âge des Esturgeons et leur longueur.

CLASSEN a utilisé pour cela l'observation directe de préparations d'écailles marginales. Mais il obtint ainsi, pour les mâles et les femelles, une série de valeurs qui ne suivaient pas une loi normale de croissance, ceci dû, sans doute, au petit nombre de ses observations. Dans de telles études, on examine les accroissements annuels des écailles marginales, puis on applique la formule de Lea-Dahl, qui donne la relation entre l'accroissement annuel du poisson et celui, en somme, d'un os de ce même poisson. C'est à l'aide de cette formule, qui n'est qu'approximative, que Classen put déterminer les longueurs moyennes des Esturgeons à leurs différents âges. Au fait, Classen, dans le classement de ses valeurs, n'a pas tenu compte d'un facteur cependant important : le sexe. Or, il n'est pas possible de faire figurer sur la méme courbe les mâles et les femelles, car les uns et les autres se développent différemment et leurs accroissements se trouvent être très distincts. Il convient donc d'établir les courbes de croissance, d'une part pour les mâles, d'autre part pour les femelles. On trouve ainsi que les longueurs moyennes pour chaque âge suivent bien une loi naturelle. Nous annexons au surplus à la présente note les graphiques obtenus. Cependant, le petit nombre de poissons étudiés (4 mâles et 9 femelles) ne permet pas de considérer ces graphiques comme définitifs, d'autant plus qu'ils ne fournissent pas de données sur les femelles de plus de 24 ans et les mâles de plus de 15 ans. Malgré tout, ils seront utiles pour illustrer ce que nous avons à dire.

En toute logique, l'influence du barrage d'Alcala del Rio, sur la reproduction de l'Esturgeon, n'a pu être remarquée qu'à partir de 1946 puisque ce barrage date de 1932 et que les femelles Esturgeons ne remontent nos cours d'eau qu'à 14 ans environ (les mâles à 11). Ce n'est donc qu'à partir de 1946 que des Esturgeons ont pu naître, à l'aval du barrage, dans des conditions non naturelles. Mais à partir de 1944, nous manquons de données sur les dimensions des Esturgeons capturés dans le Guadalquivir et nous n'avons pu en obtenir qu'en 1950. Pour cette année-là, nous possédons les fiches des individus qui entraient à l'usine de Caviar, à savoir pour l'ensemble des femelles capturées et pour la plupart des mâles.

Le manque à la reproduction devrait se manifester surtout par la diminution du nombre d'Esturgeons capturés et aussi par l'absence 
de poissons de petites dimensions. Au fait, les femelles nées à partir de 1932 à l'aval de l'Alcala del Rio avaient 14 à 18 ans lorsqu'elles ont pénétré en 1950 dans le Guadalquivir. Or, si la reproduction de 1932 à 1936 avait diminué, ce serait le poisson d'àge moindre qui, maintenant, devrait faire défaut dans la rivière, c'est-à-dire ceux de dimensions inférieures à 177 c. 4 (voir graphique). Or, on ne le constate pas, puisqu'en 1950, on a pèché 40 individus de moins de 177 c. 4 représentant $41,2 \%$ de la pêche totale, chiffre très supérieur à la moyenne des péches réalisées avant 1943. Pour les mâles, faute de données suffisantes sur leurs dimensions, on ne saurait tirer de conclusion.

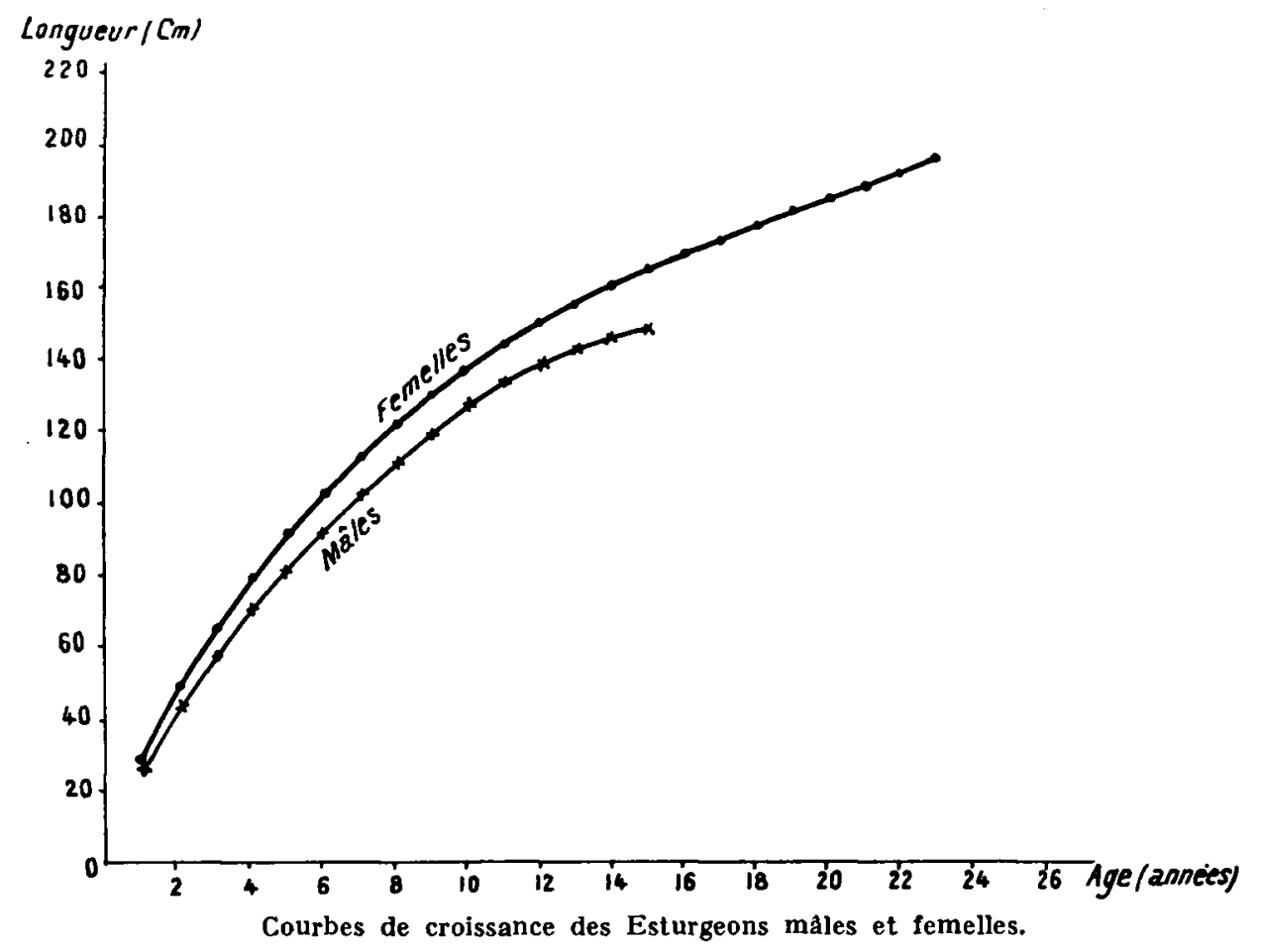

D'autre part, le nombre d'individus capturés devrait, logiquement, être moindre que durant les années précédant 1946 : effectivement, jusqu'à cette année-là, le nombre moyen d'Esturgeons capturés dans le Guadalquivir a été de 122,5 pour les femelles et de 30,3 pour les mâles. Or, en 1950, 104 femelles seulement (et 47 mâles) sont entrés dans l'usine de Caviar, ce qui laisse apparaître, pour les femelles, une diminution des captures de $15,1 \%$ par rapport à la moyenne des années précédentes.

Mais si cette diminution des captures a pu être provoquée par les diverses causes dont nous avons parlé, il serait également possible qu'elle provienne du fait que le courant d'eau douce dans la rivière n'a pas été suffisant pour inciter le poisson à remonter.

En ce qui concerne les mâles, et bien que la pêche effectuée ait dépassé la moyenne, il est prudent de n'en pas tirer de conclusions, car le contrôle n'a pas été aussi rigoureux que pour les femelles. 
De ce qui précède, il semble résulter le peu d'influence qu'ont eu les modifications du milieu vital sur la reproduction de l'Esturgeon. Mais ne serait-ce pas trop affirmer? Car si l'Esturgeon se reproduit, il le fait dans un milieu qui ne lui est pas approprié, et il se pourrait que ceci entraîne finalement une dégénérescence de l'espèce impossible à déceler dès les premières années. Au surplus, l'observation d'une seule pêche n'est pas suffisante. Et puis, il faut tenir compte que si les Esturgeons actuellement âgés de 18 ans ont pu monter à Alcala del Rio ou à La Algaba, en 1950 et durant les années précédentes, les géniteurs ne purent arriver jusqu'à ces localités et frayèrent donc dans l'eau salée, état de choses dont les conséquences ne se sont pas encore fait sentir.

Devant cet état de choses, devons-nous nous croiser les bras et attendre les résultats, plutôt que de prendre des mesures en vue de contrecarrer les conséquences possibles des faits que nous avons signalés? Et même si ces faits ne devaient pas entraîner de conséquences défavorables, n'avons-nous pas l'obligation d'augmenter le nombre des Esturgeons du Guadalquivir ? Car 104 femelles est un nombre bien faible pour la capacité biogénique d'un cours d'eau dans lequel, en une seule année, 1935, on a capturé 342 femelles?

Dans ce but, il convient d'aider l'Esturgeon à se reproduire en allant jusqu'à pratiquer la fécondation artificielle.

En outre, si l'Esturgeon n'a pas la possibilité d'accéder de lui-même à ses frayères naturelles, nous pouvons l'y aider en ouvrant les vannes du barrage lorsque la quantité d'eau retenue le permet (certaines années l'Esturgeon a pu ainsi franchir le barrage). A défaut, nous avons la faculté de pêcher des géniteurs à l'aval du barrage et de les transporter en amont dans le lac de retenue.

Encore faut-il, pour que ces moyens puissent être mis en œuvre, que les Esturgeons remontent jusqu'à Alcala del Rio, ce qui, ainsi que nous l'avons déjà dit, ne se présente pas tous les ans.

Il convient aussi de reconnaître et de " repérer " les frayères ainsi que les lieux de concentration des jeunes Esturgeons afin d'en assurer la protection.

Jusqu'à présent, on a reconnu et localisé les frayères d'Alcala et de San Salvador (à La Algaba) et on en assurera la surveillance durant les années où les Esturgeons pourront y accéder. Par contre, on ne connaît pas encore les frayères d'aval où fraient les Esturgeons en cas de manque d'eau dans la rivière. On suppose, toutefois, qu'elles doivent être situées aux abords immédiats de Séville.

Mais les meilleurs résultats, ou tout au moins les plus contrôlables, seraient ceux que l'on obtiendrait au moyen de la fécondation artificielle, bien que, dans ce domaine, nous rencontrions de grosses difficultés du fait que nous manquons de données sur les procédés utilisés dans les pays où ils auraient réussi.

En la matière, nous ne disposons, comme documentation moderne, que d'un travail de Classen, fait en collaboration avec T. Spicza kow (1),

(1) T. Classen et T. Spiczakow : Nota sobre la cria de los alevines de esturion procedentes de huevos artificialemente fecundados ", Boletin de la Real Sociedad Espanola de Historia Natur:ul, Tom. XL, 1942, Madrid. 


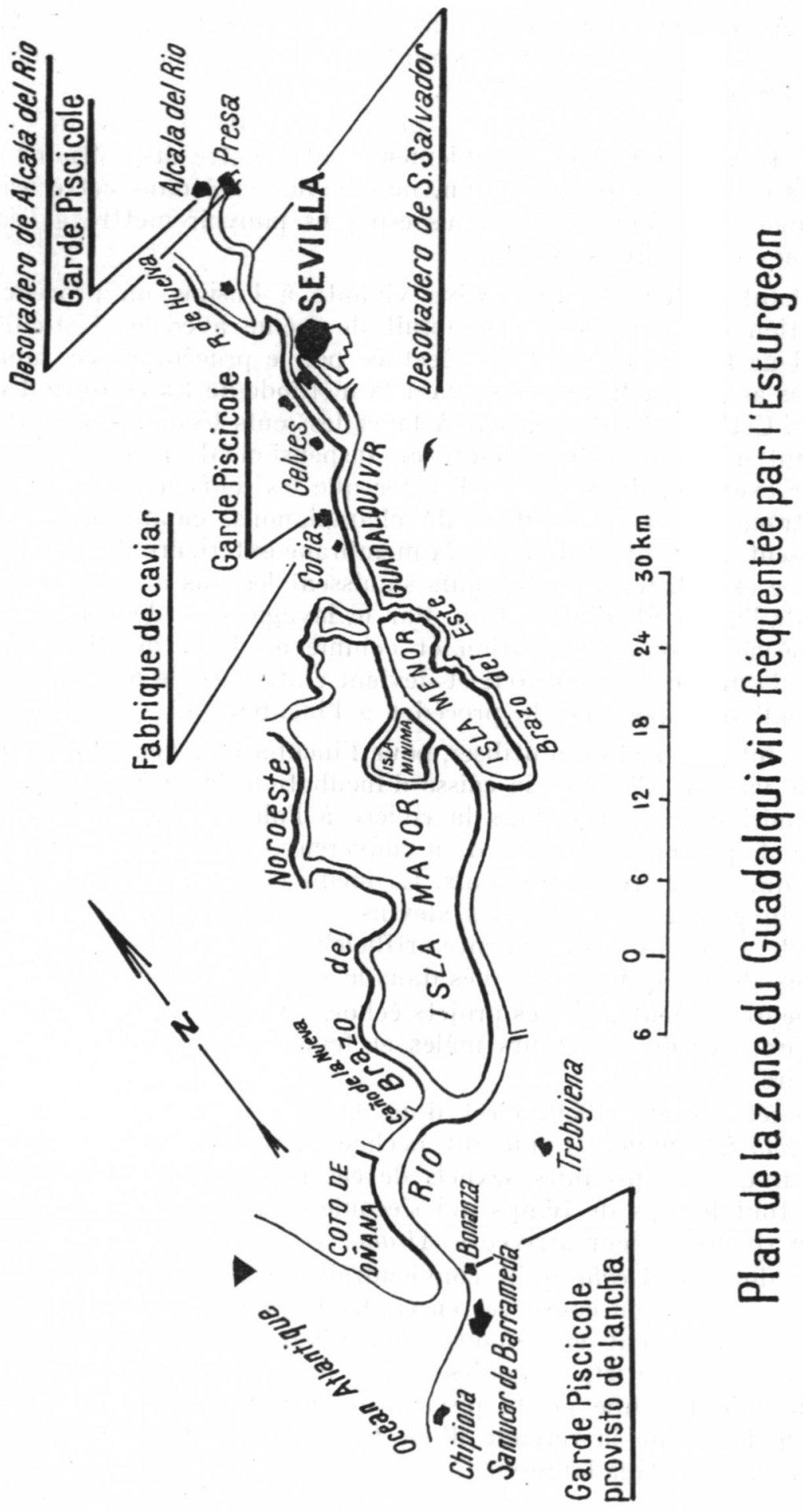


travail dans lequel il est rendu compte de quelques essais et des difficultés rencontrées et sur lequel nous avons basé nos épreuves initiales. Au fait, au cours de celles-ci, nous avons rencontré une première difficulté dans le manque de géniteurs matures.

Mettant à profit l'offre du propriétaire de l'usine de Caviar, M. José Ibarra, qui, très aimablement, mit à notre disposition tous les éléments dont nous avions besoin, nous avons créé dans cette usine un petit laboratoire dans lequel nous espérons pouvoir mettre à l'essai la technique opératoire suivante :

Sacrifiant la femelle, qui arrive vivante à l'usine, on procéderait à l'extraction des ovaires et l'on ferait de même avec les testicules des mâles. Une fois la fécondation effectuée par le procédé à sec, nous procéderions au lavage des œufs suivant la méthode de LIJw, durant quinze minutes. Cette méthode consiste à laver les œufs fécondés avec de l'eau contenant une matière abondante en suspension, de la terre glaise très fine par exemple, dans le but d'éviter que les œufs ne s'agglutinent et ne se transforment en culture de champignons, ce qui les détruirait rapidement. Au contact de l'eau, la membrane extérieure de l'œuf devient visqueuse et adhérente et les œufs s'unissent les uns aux autres ou aux parois de l'appareil d'incubation. Par le lavage avec de l'eau fangeuse, on empêche cette agglutination et comme au bout de dix ou quinze minutes la membrane se durcit et devient chitineuse, le danger disparaît. Il devient alors possible de procéder à l'incubation.

Notre intention était d'utiliser, pour l'incubation, deux types d'appareils : le vase de Chase et la caisse d'incubation de Seth-Green que nous maintiendrions flottante dans la rivière à l'aide de quelques bouchons de liège et qui serait pourvue d'un couvercle de bois pour éviter l'action directe de la lumière sur les œufs. La même caisse d'incubation pourrait être utilisée pour l'élevage des alevins et ce dans leur milieu naturel. Par contre, nous aurions alimenté artificiellement, avec des "soucoupes ", ceux des alevins qui seraient nés dans le vase de Chase.

Malheureusement tous ces projets échouèrent, car il ne fut pas possible de se procurer des géniteurs mâles et femelles dans l'état de maturité nécessaire.

En effet, durant 1950, c'est dans la partie basse du Guadalquivir (Figuerola-El Puntal...) que fût pêchée la totalité de ces Esturgeons. Or, pour que les produits sexuels de ces poissons arrivent à maturité, il faut tout le laps de temps qui s'écoule entre l'entrée des Esturgeons dans la rivière et leur arrivée à Alcala.

C'est donc à Alcala qu'il conviendrait de pêcher les reproducteurs (nous n'avons pu en conserver en captivité pendant plus de 48 heures), et c'est généralement en ce point qu'il y aurait lieu, après avoir effectué d'une manière satisfaisante les essais de reproduction artificielle, de faire fonctionner une petite pisciculture d'Esturgeons qui, tirant parti de l'eau douce de la retenue d'Alcala, assurerait la permanence de ce poisson dans le Guadalquivir. 